\title{
Non-uniform sampled scalar diffraction calculation using non-uniform fast Fourier transform
}

\author{
Tomoyoshi Shimobaba ${ }^{1}$, Takashi Kakue ${ }^{1}$, Minoru Oikawa ${ }^{1}$, Naohisa Okada ${ }^{1}$, \\ Yutaka Endo $^{1}$, Ryuji Hirayama ${ }^{1}$, and Tomoyoshi Ito ${ }^{1}$ \\ ${ }^{1}$ Graduate School of Engineering, Chiba University, 1-33 Yayoi-cho, Inage-ku, Chiba 263-8522, Japan \\ ${ }^{*}$ Corresponding author: shimobaba@faculty.chiba-u.jp
}

Compiled June 7, 2021

\begin{abstract}
Scalar diffraction calculations such as the angular spectrum method (ASM) and Fresnel diffraction, are widely used in the research fields of optics, X-rays, electron beams, and ultrasonics. It is possible to accelerate the calculation using fast Fourier transform (FFT); unfortunately, acceleration of the calculation of non-uniform sampled planes is limited due to the property of the FFT that imposes uniform sampling. In addition, it gives rise to wasteful sampling data if we calculate a plane having locally low and high spatial frequencies. In this paper, we developed non-uniform sampled ASM and Fresnel diffraction to improve the problem using the non-uniform FFT. (C) 2021 Optical Society of America
\end{abstract}

OCIS codes: (0070) Fourier optics and signal processing; (090.1760) Computer holography; (090.2870) Holographic display; (090.5694) Real-time holography; (090.1995) Digital holography.

Scalar diffraction calculations such as the angular spectrum method (ASM) and Fresnel diffraction [1] are major methods used in wide-ranging optics, especially, important tools for computer-generated holograms (CGHs), digital holography, the design of diffractive optical elements, the analysis of beam propagation and so forth. Generally, these diffractions are expressed as follows:

$$
\begin{aligned}
u_{2}\left(\boldsymbol{x}_{2}\right) & =\int u_{1}\left(\boldsymbol{x}_{1}\right) h_{z}\left(\boldsymbol{x}_{2}-\boldsymbol{x}_{1}\right) d \boldsymbol{x}_{1} \\
& =\mathcal{F}^{-1}\left[\mathcal{F}\left[u_{1}\left(\boldsymbol{x}_{1}\right)\right] H_{z}(\boldsymbol{f})\right],
\end{aligned}
$$

where $\mathcal{F}[\cdot]$ and $\mathcal{F}^{-1}[\cdot]$ are forward and inverse Fourier transforms, $u_{1}\left(\boldsymbol{x}_{1}\right)$ and $u_{2}\left(\boldsymbol{x}_{2}\right)$ are the source and destination planes, $\boldsymbol{f}=\left(f_{x}, f_{y}\right)$ is the position vector in the Fourier domain, and $z$ is the propagation distance between the source and destination planes, $h_{z}\left(\boldsymbol{x}_{2}-\boldsymbol{x}_{1}\right)$ is the impulse response, and $H_{z}(\boldsymbol{f})=\mathcal{F}\left[h_{z}\left(\boldsymbol{x}_{1}\right)\right]$ is the transfer function.

ASM is expressed as the following equations:

$$
\begin{aligned}
u_{2}\left(\boldsymbol{x}_{2}\right) & =\int \mathcal{F}\left[u_{1}\left(\boldsymbol{x}_{1}\right)\right] \exp \left(2 \pi i z \sqrt{\frac{1}{\lambda^{2}}-|\boldsymbol{f}|^{2}}\right) \times \\
& =\mathcal{F}^{-1}\left[\mathcal{F}\left[u_{1}\left(\boldsymbol{x}_{1}\right)\right] H_{z}(\boldsymbol{f})\right],
\end{aligned}
$$

where $H_{z}(\boldsymbol{f})=\exp \left(2 \pi i z \sqrt{\frac{1}{\lambda^{2}}-|\boldsymbol{f}|^{2}}\right)$ is the transfer function of ASM. In numerical calculation, it is possible to accelerate the calculation using fast Fourier transform (FFT) as follows:

$$
u_{2}\left(\boldsymbol{m}_{2}\right)=\mathrm{FFT}^{-1}\left[\operatorname{FFT}\left[u_{1}\left(\boldsymbol{m}_{1}\right)\right] H_{z, \Delta_{f}}\left(\boldsymbol{m}_{f}\right)\right],
$$

where, $\boldsymbol{m}_{1}=\left(m_{1}, n_{1}\right)$ and $\boldsymbol{m}_{2}=\left(m_{2}, n_{2}\right)$ are the position vector on the spatial domain as integer. $\boldsymbol{m}_{f}=$ $\left(m_{f}, n_{f}\right)$ is the position vector on the frequency domain as integer. The source, destination planes and frequency domain are sampled by the rates of $\Delta_{1}, \Delta_{2}$ and $\Delta_{f}$, respectively. The operators FFT[·] and $\mathrm{FFT}^{-1}[\cdot]$ are forward and inverse FFTs, respectively. The transfer function $H_{z, \Delta}\left(\boldsymbol{m}_{f}\right)[2]$ is defined by,

$$
\begin{aligned}
H_{z, \Delta}\left(\boldsymbol{m}_{f}\right)= & \exp \left(2 \pi i\left(\boldsymbol{o m}_{f}+z \sqrt{\frac{1}{\lambda^{2}}-\left|\boldsymbol{m}_{f} \Delta\right|^{2}}\right)\right) \times \\
& \operatorname{Rect}\left(\frac{m_{f}-c_{m}}{w_{m}}, \frac{n_{f}-c_{n}}{w_{n}}\right)
\end{aligned}
$$

where $\Delta$ is the sampling rate, and the offset parameter $\boldsymbol{o}=\left(o_{x}, o_{y}\right)$ is for off-axis calculation. The two-dimensional rectangle function $\operatorname{Rect}(\cdot)$ is capable of calculating long propagation of ASM, respectively. $\left(w_{m}, w_{n}\right)$ and $\left(c_{m}, c_{n}\right)$ are the band-widths and the center of the band widths, respectively. See Ref. [2] for the determination of these parameters. Likewise, we can calculate Fresnel diffraction using the following transfer function:

$$
H_{z, \Delta}\left(\boldsymbol{m}_{f}\right)=\operatorname{FFT}\left[\frac{\exp (i k z) \exp \left(i \frac{\left(\boldsymbol{m}_{1} \Delta+\boldsymbol{o}\right)^{2}}{\lambda z}\right)}{i \lambda z}\right] .
$$

Note that we have to expand the calculation size by zero-padding to $N^{\prime} \times N^{\prime}$ where $N^{\prime}=2 N$ ( $N$ is the horizontal and vertical pixel numbers of planes) in order to avoid wraparound by the circular convolution of Eq.(3). Therefore, the sampling rate $\Delta$ in the transfer functions is $\Delta=1 /\left(N^{\prime} \Delta_{1}\right)$.

However, using FFT imposes the same sampling rates on the source and destination planes $\left(\Delta_{1}=\Delta_{2}\right)$. In order to solve this restriction, scaled-Fresnel diffraction [3-7] and scaled-ASMs [8-10] that can address different sampling rates on source and destination planes were proposed. In Refs. $[3-8,10]$, chirp-z transform (a.k.a. scaled 
Fourier transform) is used instead of normal FFT. In Ref. [9], non-uniform sampling FFT (NUFFT) [11] is used. The scaling operation is, for example, useful for lensless zoomable holographic projection [12,13], digital holographic microscopy [14] and fast CGH calculation [15]. Even though scaled-ASMs can address different sampling rates on source and destination planes, each plane still requires uniform-sampling.

In this paper, we develop non-uniform sampled ASM (NU-ASM) and Fresnel diffraction (NU-FRE) to overcome the restriction using the non-uniform FFT. The uniform-sampled scalar diffractions give rise to wasteful sampling data if we calculate a plane having locally low and high spatial frequencies. In contrast, NU-ASM and NU-FRE are expected to be useful for the situation. NU-ASM is a generalized version of the scaled-ASM of Ref. [9].

First, we consider NU-ASM. We need three types of NU-ASM, that is, the first NU-ASM 1 calculates ASM from a non-uniform sampled source plane to the uniformsampled destination plane. The second NU-ASM ${ }_{2}$ is the opposite version of the first. The third, NU-ASM 3 , calculates ASM on both non-uniform sampled source and destination planes.

By applying NUFFT to Eq.(3) to a source plane, we can straightforwardly obtain NU-ASM :

$$
\begin{aligned}
u_{2}\left(\boldsymbol{m}_{2}\right) & =\operatorname{NUASM}_{1}\left[u_{1}\left(\boldsymbol{x}_{1}\right)\right] \\
& =\mathrm{FFT}^{-1}\left[\operatorname{NUFFT}_{1}\left[u_{1}\left(\boldsymbol{x}_{1}\right)\right] H_{z, \Delta}\left(\boldsymbol{m}_{f}\right)\right]
\end{aligned}
$$

where $\operatorname{NUFFT}_{1}[\cdot]$ is the type 1 of NUFFT, $\Delta=$ $1 /\left(N^{\prime} \Delta_{2}\right)$ and $u_{1}\left(\boldsymbol{x}_{1}\right)$ is the non-uniform sampled source plane. Several implementations of NUFFT were proposed. We used Greengard and Lee's NUFFT [11]. Two types of NUFFT are defined. NUFFT type 1 is defined as follows:

$$
F\left(\boldsymbol{m}_{f}\right)=\mathrm{NUFFT}_{1}\left[f\left(\boldsymbol{x}_{1}^{\prime}\right)\right]=\sum_{\boldsymbol{x}_{1}^{\prime}} f\left(\boldsymbol{x}_{1}^{\prime}\right) \exp \left(-i \pi \boldsymbol{m}_{f} \boldsymbol{x}_{1}^{\prime}\right),
$$

where, $\boldsymbol{m}_{f}$ is uniform sampled with the integer value on the frequency domain. Practically, the right-side of the equation is accelerated by FFT, gridding algorithm and deconvolution [11].

Due to the NUFFT property, we need to normalize the coordinate of the source plane as shown in Fig.1. The original coordinate system of the source plane $\boldsymbol{x}_{1}$ is defined as $\boldsymbol{x}_{1} \in[-N / 2, N / 2) \times[-N / 2, N / 2)$ as shown in Fig.1(a). The NUFFT requires the range of the coordinate, $\boldsymbol{x}_{1}^{\prime} \in[0,2 \pi) \times[0,2 \pi)$ as shown in Fig.1(b). Therefore, we convert the coordinate system by $\boldsymbol{x}_{1}^{\prime}=$ $2 \pi\left(\boldsymbol{o}^{\prime}+\boldsymbol{x}_{1}\right) / N$ where $\boldsymbol{o}^{\prime}=(N / 2, N / 2)$.

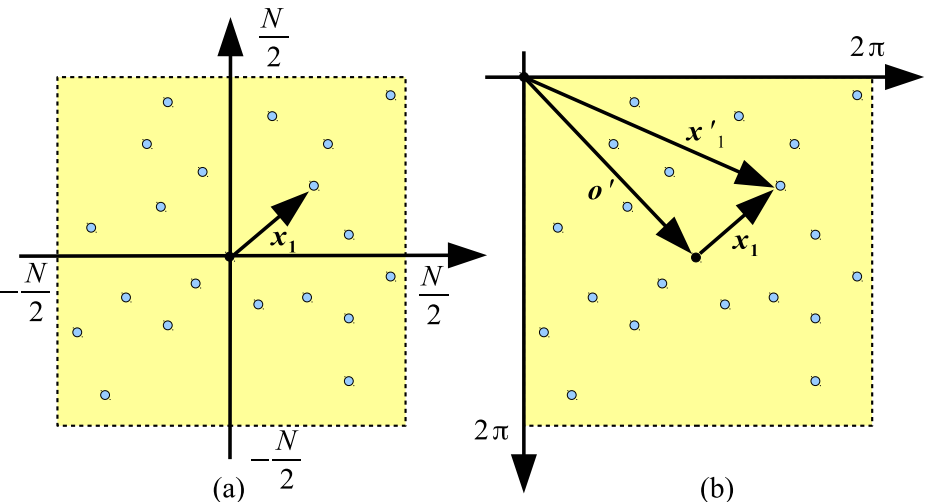

Fig. 1. Coordinate conversion for NUFFT. (a) coordinate system of the original plane (b) coordinate system of the plane for NUFFT. Blue circles indicate non-uniform sampled points.

NUFFT type 2 is defined as follows:

$f\left(\boldsymbol{x}_{2}^{\prime}\right)=\operatorname{NUFFT}_{2}\left[F\left(\boldsymbol{m}_{f}\right)\right]=\sum_{\boldsymbol{m}_{f}} F\left(\boldsymbol{m}_{f}\right) \exp \left(i \pi \boldsymbol{m}_{f} \boldsymbol{x}_{2}^{\prime}\right)$,

where $f\left(\boldsymbol{x}_{2}^{\prime}\right)$ is the non-uniform sampled plane. Note that we need to normalize the coordinate of the destination plane for the same reason as that of $\mathrm{NUFFT}_{1}$. For more details, see [11].

Likewise, $\mathrm{NU}-\mathrm{ASM}_{2}$ is obtained as follows:

$$
\begin{aligned}
u_{2}\left(\boldsymbol{x}_{2}\right) & =\operatorname{NUASM}_{2}\left[u_{1}\left(\boldsymbol{m}_{1}\right)\right], \\
& =\operatorname{NUFFT}_{2}\left[\operatorname{FFT}\left[u_{1}\left(\boldsymbol{m}_{1}\right)\right] H_{z, \Delta}\left(\boldsymbol{m}_{f}\right)\right],
\end{aligned}
$$

where $\Delta=1 /\left(N^{\prime} \Delta_{1}\right) \cdot \boldsymbol{m}_{1}$ and $\boldsymbol{m}_{f}$ are uniform sampled. $\boldsymbol{x}_{2}$ is non-uniform sampled and the range is $\boldsymbol{x}_{2} \in$ $[-N / 2, N / 2) \times[-N / 2, N / 2)$. The $\mathrm{NUFFT}_{2}$ requires the range of the coordinate, $\boldsymbol{x}_{2}^{\prime} \in[0,2 \pi) \times[0,2 \pi)$. Therefore, we convert the coordinate system by $\boldsymbol{x}_{2}^{\prime}=2 \pi\left(\boldsymbol{o}^{\prime}+\boldsymbol{x}_{2}\right) / N$.

We obtain NU-ASM 3 by combining NU-ASM 1 and NU-ASM ${ }_{2}$. In addition, using Eq.(5) instead of the transfer functions of NU-ASM, we can straightforwardly derive three types of NU-FRE, that is, NU-FRE 1 , NU$\mathrm{FRE}_{2}$ and NU-FRE . $_{3}$

Let us compare the NU-ASM and NU-FRE with Rayleigh-Sommerfeld (RS) diffraction. RS diffraction is rigorous scalar diffraction and is expressed as,

$$
u_{2}\left(\boldsymbol{x}_{2}\right)=\frac{1}{2 \pi} \iint u_{1}\left(\boldsymbol{x}_{1}\right)\left\{\frac{z}{r}(1-i k r) \frac{\exp (i k r)}{r^{2}}\right\} d \boldsymbol{x}_{1},
$$

where $k$ is the wave number and $r=\sqrt{\left|\boldsymbol{x}_{1}-\boldsymbol{x}_{2}\right|^{2}+z^{2}}$. $\mathrm{RS}$ diffraction with non-uniform sampled planes is calculated by directly numerical integration, which has the complexity of $O\left(N^{4}\right)$.

We use Fig. 2 as the non-uniform sampled source plane with $256 \times 256$ pixels. The image "Lenna" is the am- 

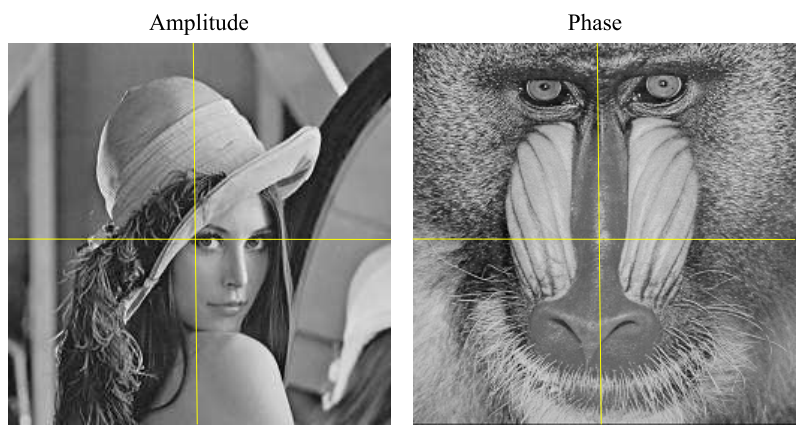

Fig. 2. Source plane with $256 \times 256$ pixels. "Lenna" as the amplitude distribution of the source plane, and the image "Mandrill" as the phase distribution where the pixel value 0 corresponds to the phase value $-\pi / 2$ and the pixel value 255 corresponds to the phase value $+\pi / 2$.

plitude distribution of the source plane, and the image "Mandrill" is the phase distribution where the pixel value 0 corresponds to the phase value $-\pi / 2$ and the pixel value 255 corresponds to the phase value $+\pi / 2$. The calculation condition is as follows: the wavelength is $500 \mathrm{~nm}$ and the sampling rate on the uniform-sampled destination plane is $\Delta_{2}=5 \mu \mathrm{m}$. The source plane is nonuniform-sampled, where the sampling rates on the first to fourth quadrants are $0.9 \Delta_{2}, 0.8 \Delta_{2}, 0.7 \Delta_{2}$ and $\Delta_{2}$, respectively.

Figure 3(a) and (b) are the amplitude and phase on the diffracted result by RS diffraction at $z=8 \mathrm{~mm}$. Figure 3(c) and (d) are the amplitude and phase on the diffracted result by $\mathrm{NU}_{-} \mathrm{ASM}_{1}$. In Fig. 4, we measure the error of NU-ASM 1 and NU-FRE 1 to RS diffraction, which is the criteria, by the signal-to-noise ratio (SNR). At short distance propagation, the SNR of NU-ASM is good quality, while that of NU-FRE 1 is poor quality. Whereas, at long distance propagation, the SNR of $\mathrm{NU}_{-} \mathrm{ASM}_{1}$ is decreased because the band-limited transfer function of Eq.(4) has a small area, which is pointed out by Ref. [16]. While, the SNR of NU-FRE ${ }_{1}$ is increased. The average calculation time of the NU-ASM 1 is about $46 \mathrm{~ms}$, while that of RS diffraction is about $76 \mathrm{~s}$. We use an Intel Core i7-2600S CPU and eight CPU threads, and multi-thread version of FFTW [17] as the FFT library.

Figure 5 shows diffracted results from a uniformsampled source plane to non-uniform-sampled destination plane by RS diffraction and NU-ASM 2 . We use Fig.2 as the uniform-sampled source plane at the sampling rate $\Delta_{1}=5 \mu \mathrm{m}$. The destination plane is non-uniformsampled, where the sampling rates on the first to fourth quadrants are $0.9 \Delta_{1}, 0.8 \Delta_{1}, 0.7 \Delta_{1}$ and $\Delta_{1}$, respectively. Figure 5(a) and (b) are the amplitude and phase on the diffracted result by RS diffraction at $z=8 \mathrm{~mm}$. Figure 5(c) and (d) are the amplitude and phase on the diffracted result by $\mathrm{NU}-\mathrm{ASM}_{2}$. The $\mathrm{SNR}$ is maintained at approximately $32 \mathrm{~dB}$.

We conclude this work. This paper proposes the nonuniform sampled scalar diffractions, NU-ASM and NU- (a)

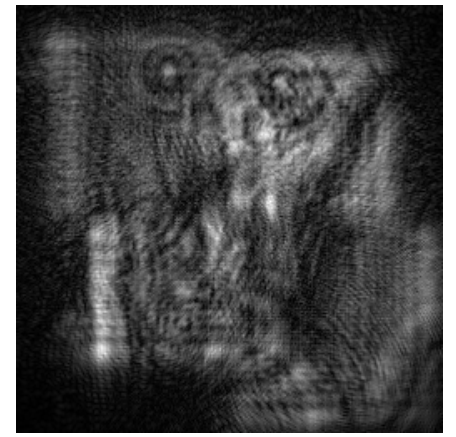

(c)

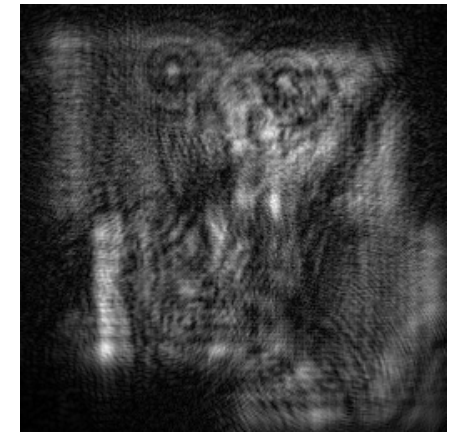

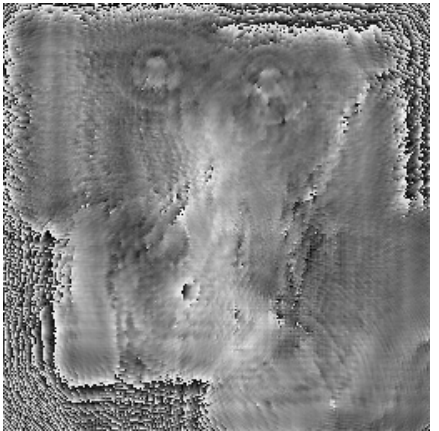

(b)

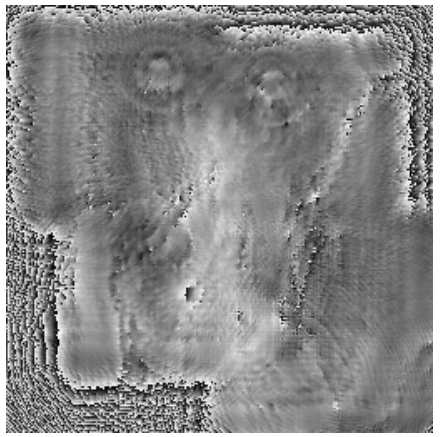

(d)
Fig. 3. Diffracted results of the amplitude and phase distribution by RS diffraction and NU-ASM. (a) amplitude by RS diffraction (b) phase by RS diffraction (c) amplitude by NU-ASM (d) phase by NU-ASM.

FRE, based on NUFFT. These diffractions are categorized into three types: type 1 is the diffraction from a non-uniform sampled source plane to a uniform sampled destination plane, the type 2 is the opposite situation of type 1; and type 3 is the diffraction between a non-uniform sampled source and destination planes. NU-ASM is suitable for the short propagation, while NU-FRE are suitable for the long propagation. In addition, the calculation times of NU-ASM and NU-FRE is faster than RS diffraction. In future, we will attempt to apply the non-uniform sampled scalar diffractions to CGH calculation, the design of optical elements, and optical encryption and watermarking. These diffraction calculations will be provided in our open-source library: $\mathrm{CWO}++$ library [18].

This work is supported by the Ministry of Internal Affairs and Communications, Strategic Information and Communications R\&D Promotion Programme (SCOPE)(09150542), Japan Society for the Promotion of Science (JSPS) KAKENHI (Young Scientists (B) 23700103) 2011, and the NAKAJIMA FOUNDATION.

\section{References}

1. J.W.Goodman, "Introduction to Fourier Optics (3rd ed.)," Robert \& Company (2005).

2. K. Matsushima, "Shifted angular spectrum method for off-axis numerical propagation," Opt. Express 18, 18453-18463 (2010). 


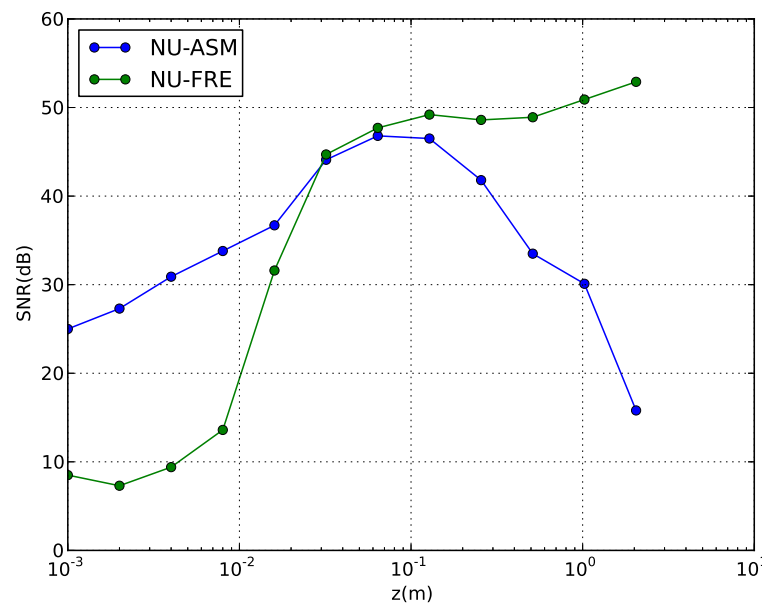

Fig. 4. Errors of NU-ASM 1 and NU-FRE $E_{1}$ to RS diffraction by the signal-to-noise ratio (SNR).

3. L. Yaroslavsky, "Optical transforms in digital holography," Proc. SPIE Holography 2005: International Conference on Holography, Optical Recording, and Processing of Information, 6252, 625216 (2006).

4. R. P. Muffoletto, J. M. Tyler, and J. E. Tohline, "Shifted Fresnel diffraction for computational holography," Opt. Express 15, 5631-5640 (2007).

5. J. F. Restrepo and J. G. -Sucerquia, "Magnified reconstruction of digitally recorded holograms by FresnelBluestein transform," Appl. Opt. 49, 6430-6435 (2010)

6. L. Bilevich and L. Yaroslavsky, "Fast DCT-based image convolution algorithms and application to image resampling and hologram reconstruction," Proc. SPIE 7724, $77240 \mathrm{~N}(2010)$.

7. T. Shimobaba, T. Kakue, N. Okada, M. Oikawa, Y. Yamaguchi, and T. Ito, "Aliasing-reduced Fresnel diffraction with scale and shift operations," J.Opt. 15075405 (2013).

8. S. Odate, C. Koike, H. Toba, T. Koike, A. Sugaya, K. Sugisaki, K. Otaki, and K. Uchikawa, "Angular spectrum calculations for arbitrary focal length with a scaled convolution," Opt. Express 19, 14268-14276 (2011).

9. T. Shimobaba, K. Matsushima, T. Kakue, N. Masuda, and T. Ito, "Scaled angular spectrum method," Opt. Lett. 37, 4128-4130 (2012).

10. X. Yu, T. Xiahui, Q. Yingxiong, P. Hao, and W. Wei, "Band-limited angular spectrum numerical propagation method with selective scaling of observation window size and sample number," J. Opt. Soc. Am. A 29, 2415-2420 (2012).

11. L. Greengard and J. Y. Lee, "Accelerating the nonuniform fast Fourier transform, " SIAM Rev. 46, 443-454 (2004).

12. T. Shimobaba, T. Kakue, N. Masuda and T. Ito, "Numerical investigation of zoomable holographic projection without a zoom lens," J. Soc. Info. Disp. 20, 533-538 (2012).

13. T. Shimobaba, M. Makowski, T. Kakue, M. Oikawa, N. Okada, Y. Endo, R. Hirayama, and T. Ito, "Lens-

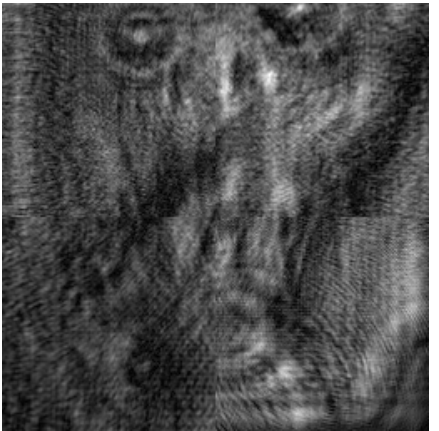

(a)

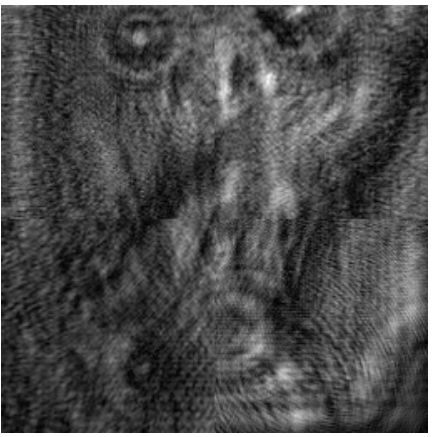

(c)

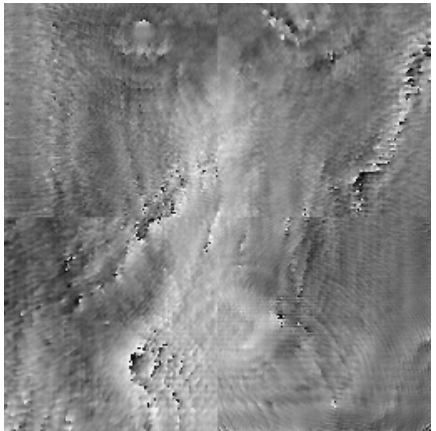

(b)

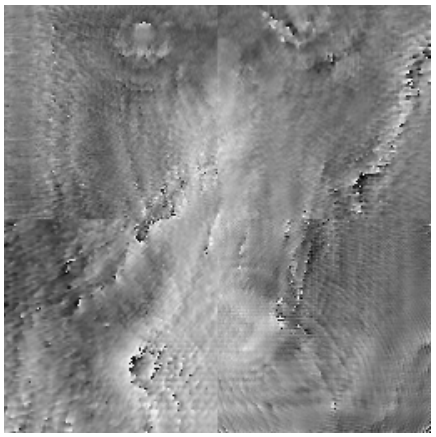

(d)
Fig. 5. Diffracted results of the amplitude and phase distribution by RS diffraction and NU-ASM. (a) amplitude by RS diffraction (b) phase by RS diffraction (c) amplitude by NU-ASM (d) phase by NU-ASM.

less zoomable holographic projection using scaled Fresnel diffraction," Opt. Express (accepted).

14. T. Shimobaba, N. Masuda, Y. Ichihashi, and T. Ito, "Real-time digital holographic microscopy observable in multi-view and multi-resolution," J. Opt. 12, 065402 (2010).

15. J. Weng, T. Shimobaba, N. Okada, H. Nakayama, M. Oikawa, N. Masuda, and T. Ito, , "Generation of realtime large computer generated hologram using wavefront recording method," Opt. Express 20, 4018-4023 (2012).

16. X. Yu, T. Xiahui, Q. Y. Xiong, P. Hao, and W. Wei, "Wide-window angular spectrum method for diffraction propagation in far and near field," Opt. Lett. 37, 49434945 (2012).

17. M. Frigo and S. G. Johnson, "FFTW: An adaptive software architecture for the FFT," in Proc. 1998 IEEE Intl. Conf. on Acoustics, Speech, and Signal Processing (Institute of Electrical and Electronics Engineers, New York, 1998), 1381-1384.

18. T. Shimobaba, J. Weng, T. Sakurai, N. Okada, T. Nishitsuji, N. Takada, A. Shiraki, N. Masuda, and T. Ito, Comput. Phys. Commun. 183, 1124-1138 (2012). 